\title{
História das placas de formatura da Faculdade de Medicina da Universidade Federal do Ceará
}

\author{
I) A Faculdade de Medicina da UFC
}

O curso de medicina do Ceará foi criado no dia 12 de maio de 1948. Seus fundadores líderes foram Jurandir Picanço, Waldemar de Alcântara, José Carlos Ribeiro, Newton Gonçalves e Walter de Moura Cantídio. Em 27 de março de 1951, o Presidente Getúlio Vargas reconheceu o curso, que passou a se chamar Faculdade de Medicina do Ceará. Dos dez alunos que iniciaram o curso em 1948, somente três colaram grau na primeira turma, em dezembro de 1953. Um dos três concludentes, Raimundo Hélio Cirino Bessa, veio a se tornar posteriormente professor de cardiologia dessa faculdade. No ano de 1954, a faculdade passou a pertencer à Universidade Federal do Ceará (UFC). Em 1973, uma reforma universitária tornou a Faculdade de Medicina um curso do Centro de Ciências da Saúde (CCS). Mas em 1997, numa gestão do Prof Elias Salomão Boutala, o curso de Medicina da UFC foi desvinculado do CCS e voltou a ser chamado de Faculdade de Medicina da UFC (FM-UFC).

II) As placas de formatura

Até o ano de 1970, entrava apenas uma turma por ano através de seu concurso vestibular. A partir de 1971, passaram a entrar duas turmas por ano, o que acontece até hoje. A turma que entrou no segundo semestre de 1971 colou grau no final do primeiro semestre de 1977. Sendo assim, desde 1977, temos turma 1977.1, 1977.2, etc. No entanto, constava no arquivo da coordenação do curso da FM-UFC duas turmas anuais em 1973, 1974, 1975 e 1976, quando, na realidade, até 1976, só havia uma turma por ano.

Passou a se colocar o número ordinal nas placas a partir da turma de 1988.1. Baseado nas informações dos arquivos do curso, a comissão de formatura dessa turma escreveu na placa que essa era a $51^{a}$ turma, quando na verdade essa é a $46^{a}$ turma. A partir dessa placa, então, todas as outras que tem o número ordinal até a turma de 2019.1 estão com esta informação incorreta. Devido a uma prolongada greve de professores, não houve turma se formando no primeiro semestre de 1985.

Alguns anos atrás foi feita uma reforma no prédio da biblioteca da Faculdade de Medicina da UFC e todas as placas de formatura que estavam afixadas em suas paredes foram retiradas com a intenção de serem recolocadas em algum lugar depois da reforma. A reforma foi encerrada e as placas não foram recolocadas durante anos. Há cerca de dois anos, a Professora Valéria Goes Ferreira, então diretora da Faculdade de Medicina, determinou que se localizassem essas placas e que elas fossem novamente afixadas. Surgiram quinze placas, mas infelizmente não reapareceu a placa da minha turma, 1986.2. A Professora Valéria escolheu um excelente local para recolocação dessas placas, as paredes externas do Departamento de Medicina Legal e Patologia (DPML). Até parece, que essas colunas tinham sido construídas para receber tais placas. Depois deste fato, me veio o desejo de fazer um inventário de todas as placas, fotografá-las e descrever o lugar onde elas estão afixadas (Tabela 1). Elas são em número de 82. O Hospital Universitário Walter Cantídio abriga o maior número de placas (53), distribuídas em nove locais do hospital. No DPML, além das colunas nas paredes externas, há uma placa na escada que dá acesso ao andar superior, totalizando 16 placas. O Departamento de Morfologia abriga sete placas. Existem três placas nos anexos da biblioteca. O bloco didático I, prédio da biomedicina e a Maternidade Escola abrigam uma placa, cada. A placa da turma mais antiga é a de 1962, mas esta não é uma placa original da época da colação de grau desta turma. Trata-se de placa comemorativa aos 30 anos de formatura desta turma. O mesmo acontece com as placas das turmas de 1963, 1964, 1968, 1974 e 1975. A placa original da época da formatura mais antiga que há é da turma de 1976.

Agradecimento aos meus amigos Dr. José Eloy da Costa Filho, pelas sugestões, e Prof. Dr. Armênio Santos, pelo estímulo a escrever este artigo.

Tabela 1. Números das turmas e locais das placas de formatura.

\begin{tabular}{|c|c|c|c|c|c|c|c|}
\hline \multicolumn{8}{|c|}{ Placas de formatura da FM - UFC } \\
\hline \multirow{2}{*}{ Ano/Semestre } & \multicolumn{2}{|c|}{$\mathrm{N}^{\mathrm{o}}$ Turma } & \multirow{2}{*}{ Loc. } & \multirow{2}{*}{ Ano/Semestre } & \multicolumn{2}{|c|}{$\mathrm{N}^{\mathrm{o}}$ Turma } & \multirow{2}{*}{ Loc. } \\
\hline & Real & Placa & & & Real & Placa & \\
\hline 1962 & $10^{\mathrm{a}}$ & & A & 1998.2 & $67^{\mathrm{a}}$ & $72^{\mathrm{a}}$ & $\mathrm{F}$ \\
\hline 1963 & $11^{\mathrm{a}}$ & & A & 1999.2 & $69^{\mathrm{a}}$ & & B \\
\hline
\end{tabular}

Continua. 
Continuação.

Tabela 1. Números das turmas e locais das placas de formatura.

\begin{tabular}{|c|c|c|c|c|c|c|c|}
\hline \multicolumn{8}{|c|}{ Placas de formatura da FM - UFC } \\
\hline \multirow{2}{*}{ Ano/Semestre } & \multicolumn{2}{|c|}{$\mathrm{N}^{\mathrm{o}}$ Turma } & \multirow{2}{*}{ Loc. } & \multirow{2}{*}{ Ano/Semestre } & \multicolumn{2}{|c|}{$\mathrm{N}^{\mathrm{o}}$ Turma } & \multirow{2}{*}{ Loc. } \\
\hline & Real & Placa & & & Real & Placa & \\
\hline 1964 & $12^{\mathrm{a}}$ & & M & 2000.1 & $70^{\mathrm{a}}$ & & $\mathrm{L}$ \\
\hline 1968 & $16^{\mathrm{a}}$ & & M & 2000.2 & $71^{\mathrm{a}}$ & & $\mathrm{H}$ \\
\hline 1974 & $22^{\mathrm{a}}$ & & $\mathrm{A}$ & 2001.1 & $72^{\mathrm{a}}$ & & $\mathrm{H}$ \\
\hline 1975 & $23^{\mathrm{a}}$ & & $\mathrm{N}$ & 2001.2 & $73^{\mathrm{a}}$ & $78^{\mathrm{a}}$ & $\mathrm{H}$ \\
\hline 1976 & $24^{\mathrm{a}}$ & & M & 2002.1 & $74^{\mathrm{a}}$ & $79^{\mathrm{a}}$ & $\mathrm{H}$ \\
\hline 1977.1 & $25^{\mathrm{a}}$ & & M & 2002.2 & $75^{\mathrm{a}}$ & & $\mathrm{E}$ \\
\hline 1977.2 & $26^{\mathrm{a}}$ & & $\mathrm{E}$ & 2003.1 & $76^{\mathrm{a}}$ & $81^{\mathrm{a}}$ & $\mathrm{E}$ \\
\hline 1978.1 & $27^{\mathrm{a}}$ & & M & 2003.2 & $77^{\mathrm{a}}$ & $82^{\mathrm{a}}$ & $\mathrm{H}$ \\
\hline 1978.2 & $28^{\mathrm{a}}$ & & $\mathrm{P}$ & 2004.1 & $78^{\mathrm{a}}$ & $83^{\mathrm{a}}$ & $\mathrm{H}$ \\
\hline 1979.2 & $30^{\mathrm{a}}$ & & $\mathrm{H}$ & 2004.2 & $79^{\mathrm{a}}$ & $84^{\mathrm{a}}$ & $\mathrm{F}$ \\
\hline 1980.2 & $32^{\mathrm{a}}$ & & $\mathrm{F}$ & 2005.1 & $80^{\mathrm{a}}$ & $85^{\mathrm{a}}$ & B \\
\hline 1981.2 & $34^{\mathrm{a}}$ & & M & 2005.2 & $81^{\mathrm{a}}$ & $86^{\mathrm{a}}$ & $\mathrm{H}$ \\
\hline 1982.1 & $35^{\mathrm{a}}$ & & B & 2006.1 & $82^{\mathrm{a}}$ & $87^{\mathrm{a}}$ & $\mathrm{H}$ \\
\hline 1982.2 & $36^{\mathrm{a}}$ & & M & 2006.2 & $83^{\mathrm{a}}$ & $88^{\mathrm{a}}$ & I \\
\hline 1983.1 & $37^{\mathrm{a}}$ & & M & 2007.1 & $84^{\mathrm{a}}$ & $89^{\mathrm{a}}$ & $\mathrm{C}$ \\
\hline 1983.2 & $38^{\mathrm{a}}$ & & M & 2007.2 & $85^{\mathrm{a}}$ & $90^{\mathrm{a}}$ & B \\
\hline 1984.1 & $39^{\mathrm{a}}$ & & M & 2008.1 & $86^{\mathrm{a}}$ & $91^{\mathrm{a}}$ & $\mathrm{C}$ \\
\hline 1984.2 & $40^{\mathrm{a}}$ & & B & 2008.2 & $87^{\mathrm{a}}$ & & $\mathrm{H}$ \\
\hline 1985.2 & $41^{\mathrm{a}}$ & & M & 2009.1 & $88^{\mathrm{a}}$ & & $\mathrm{H}$ \\
\hline 1986.1 & $42^{\mathrm{a}}$ & & $\mathrm{N}$ & 2009.2 & $89^{\mathrm{a}}$ & $94^{\mathrm{a}}$ & B \\
\hline 1987.2 & $45^{\mathrm{a}}$ & & M & 2010.1 & $90^{\mathrm{a}}$ & $95^{\mathrm{a}}$ & $\mathrm{C}$ \\
\hline 1988.1 & $46^{\mathrm{a}}$ & $51^{\mathrm{a}}$ & M & 2010.2 & $91^{\mathrm{a}}$ & $96^{\mathrm{a}}$ & B \\
\hline 1988.2 & $47^{\mathrm{a}}$ & $52^{\mathrm{a}}$ & M & 2011.1 & $92^{\mathrm{a}}$ & $97^{\mathrm{a}}$ & $\mathrm{C}$ \\
\hline 1989.1 & $48^{\mathrm{a}}$ & $53^{\mathrm{a}}$ & $\mathrm{J}$ & 2011.2 & $93^{\mathrm{a}}$ & $98^{\mathrm{a}}$ & G \\
\hline 1989.2 & $49^{\mathrm{a}}$ & $54^{\mathrm{a}}$ & $\mathrm{O}$ & 2012.1 & $94^{\mathrm{a}}$ & & B \\
\hline 1990.1 & $50^{\mathrm{a}}$ & $55^{\mathrm{a}}$ & $\mathrm{O}$ & 2012.2 & $95^{\mathrm{a}}$ & $100^{\mathrm{a}}$ & $\mathrm{D}$ \\
\hline 1990.2 & $51^{\mathrm{a}}$ & $56^{\mathrm{a}}$ & $\mathrm{O}$ & 2013.1 & $96^{\mathrm{a}}$ & & $\mathrm{G}$ \\
\hline 1991.1 & $52^{\mathrm{a}}$ & & $\mathrm{O}$ & 2013.2 & $97^{\mathrm{a}}$ & $102^{\mathrm{a}}$ & G \\
\hline 1991.2 & $53^{\mathrm{a}}$ & & $\mathrm{O}$ & 2014.1 & $98^{\mathrm{a}}$ & & G \\
\hline 1992.1 & $54^{\mathrm{a}}$ & $59^{\mathrm{a}}$ & $\mathrm{O}$ & 2014.2 & $99^{\mathrm{a}}$ & $104^{\mathrm{a}}$ & I \\
\hline 1992.2 & $55^{\mathrm{a}}$ & $60^{\mathrm{a}}$ & M & 2015.1 & $100^{\mathrm{a}}$ & $105^{\mathrm{a}}$ & G \\
\hline 1993.2 & $57^{\mathrm{a}}$ & $62^{\mathrm{a}}$ & $\mathrm{O}$ & 2015.2 & $101^{\mathrm{a}}$ & $106^{\mathrm{a}}$ & $\mathrm{H}$ \\
\hline 1994.1 & $58^{\mathrm{a}}$ & & E & 2016.1 & $102^{\mathrm{a}}$ & & I \\
\hline 1994.2 & $59^{\mathrm{a}}$ & $64^{\mathrm{a}}$ & Q & 2016.2 & $103^{\mathrm{a}}$ & $108^{\mathrm{a}}$ & G \\
\hline 1995.1 & $60^{\mathrm{a}}$ & & $\mathrm{E}$ & 2017.1 & $104^{\mathrm{a}}$ & $109^{\mathrm{a}}$ & $\mathrm{H}$ \\
\hline
\end{tabular}

Continua. 
Conclusão.

Tabela 1. Números das turmas e locais das placas de formatura.

\begin{tabular}{|c|c|c|c|c|c|c|c|}
\hline \multicolumn{8}{|c|}{ Placas de formatura da FM - UFC } \\
\hline \multirow{2}{*}{ Ano/Semestre } & \multicolumn{2}{|c|}{$\mathrm{N}^{\mathrm{o}}$ Turma } & \multirow{2}{*}{ Loc. } & \multirow{2}{*}{ Ano/Semestre } & \multicolumn{2}{|c|}{$\mathrm{N}^{\mathrm{o}}$ Turma } & \multirow{2}{*}{ Loc. } \\
\hline & Real & Placa & & & Real & Placa & \\
\hline 1995.2 & $61^{\mathrm{a}}$ & $66^{\mathrm{a}}$ & $\mathrm{N}$ & 2017.2 & $105^{\mathrm{a}}$ & $110^{\mathrm{a}}$ & $\mathrm{D}$ \\
\hline 1996.1 & $62^{\mathrm{a}}$ & $67^{\mathrm{a}}$ & $\mathrm{E}$ & 2018.1 & $106^{\mathrm{a}}$ & $111^{\mathrm{a}}$ & I \\
\hline 1997.2 & $65^{\mathrm{a}}$ & $70^{\mathrm{a}}$ & $\mathrm{F}$ & 2018.2 & $107^{\mathrm{a}}$ & $112^{\mathrm{a}}$ & $\mathrm{H}$ \\
\hline 1998.1 & $66^{\mathrm{a}}$ & & $\mathrm{F}$ & 2019.1 & $108^{\mathrm{a}}$ & $113^{\mathrm{a}}$ & $\mathrm{H}$ \\
\hline \multicolumn{8}{|c|}{ Localização das placas } \\
\hline $\mathbf{A}$ & & \multicolumn{5}{|c|}{ Saguão da entrada principal do Hospital Walter Cantídio } & \\
\hline B & & \multicolumn{4}{|c|}{ Corredor que segue até o segundo saguão } & & \\
\hline $\mathbf{C}$ & & \multicolumn{3}{|c|}{ Saguão: onde se inicia a escada } & & & \\
\hline D & & \multicolumn{2}{|c|}{ Escada para 1o andar } & & & & \\
\hline $\mathbf{E}$ & & \multicolumn{2}{|c|}{ Saguão do 10 andar } & & & & \\
\hline $\mathbf{F}$ & & \multicolumn{4}{|c|}{ Corredor que dá acesso à Clínica Médica I } & & \\
\hline G & & \multicolumn{4}{|c|}{ Corredores que dão acesso ao prédio da cirurgia } & & \\
\hline $\mathbf{H}$ & & \multicolumn{3}{|c|}{ Corredor vizinho ao centro cirúrgico } & & & \\
\hline I & & \multicolumn{3}{|c|}{ Corredor ao lado da radiologia } & & & \\
\hline $\mathbf{J}$ & & \multicolumn{3}{|c|}{ Térreo do bloco didático I } & & & \\
\hline $\mathbf{L}$ & & \multicolumn{6}{|c|}{ Departamento de Patologia e Medicina Legal (DPML), escada } \\
\hline $\mathbf{M}$ & & \multicolumn{3}{|c|}{ Paredes externas do DPML } & & & \\
\hline $\mathbf{N}$ & & \multicolumn{2}{|c|}{ Anexos da Biblioteca } & & & & \\
\hline $\mathbf{O}$ & & \multicolumn{3}{|c|}{ Departamento de Mofologia } & & & \\
\hline $\mathbf{P}$ & & \multicolumn{2}{|c|}{ Biomedicina } & & & & \\
\hline $\mathbf{Q}$ & & MEAC & & & & & \\
\hline
\end{tabular}

Figura 1. Placas de formatura da FM - UFC.
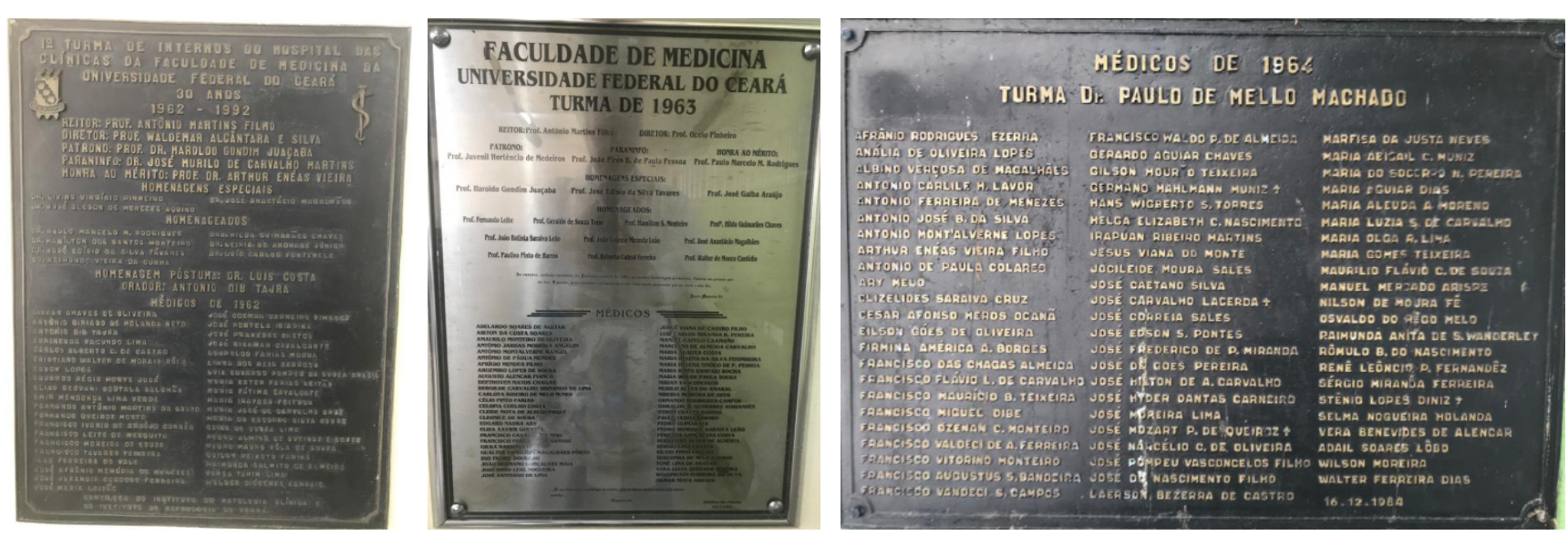
Figura 1. Placas de formatura da FM - UFC.
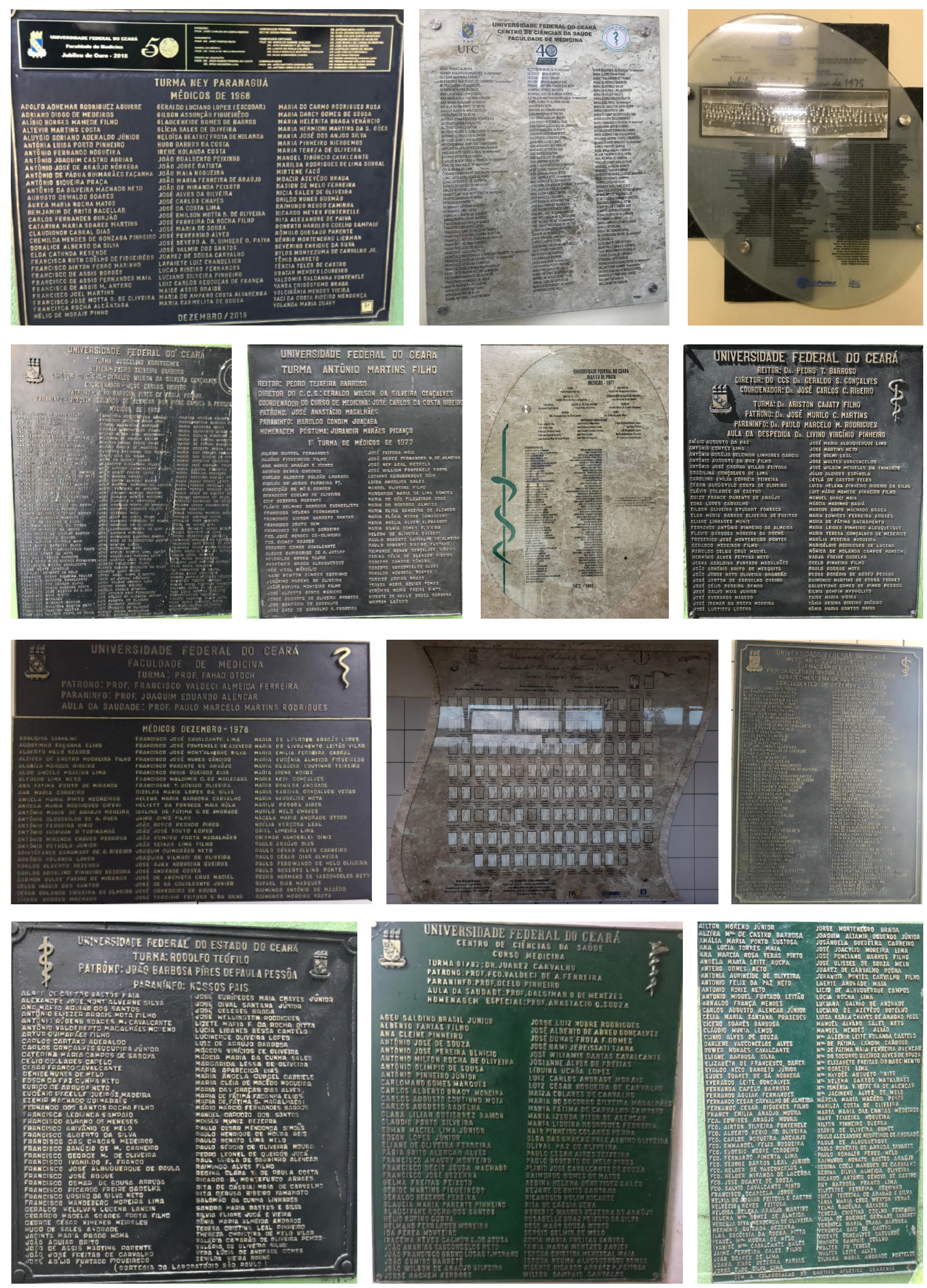
Figura 1. Placas de formatura da FM - UFC.
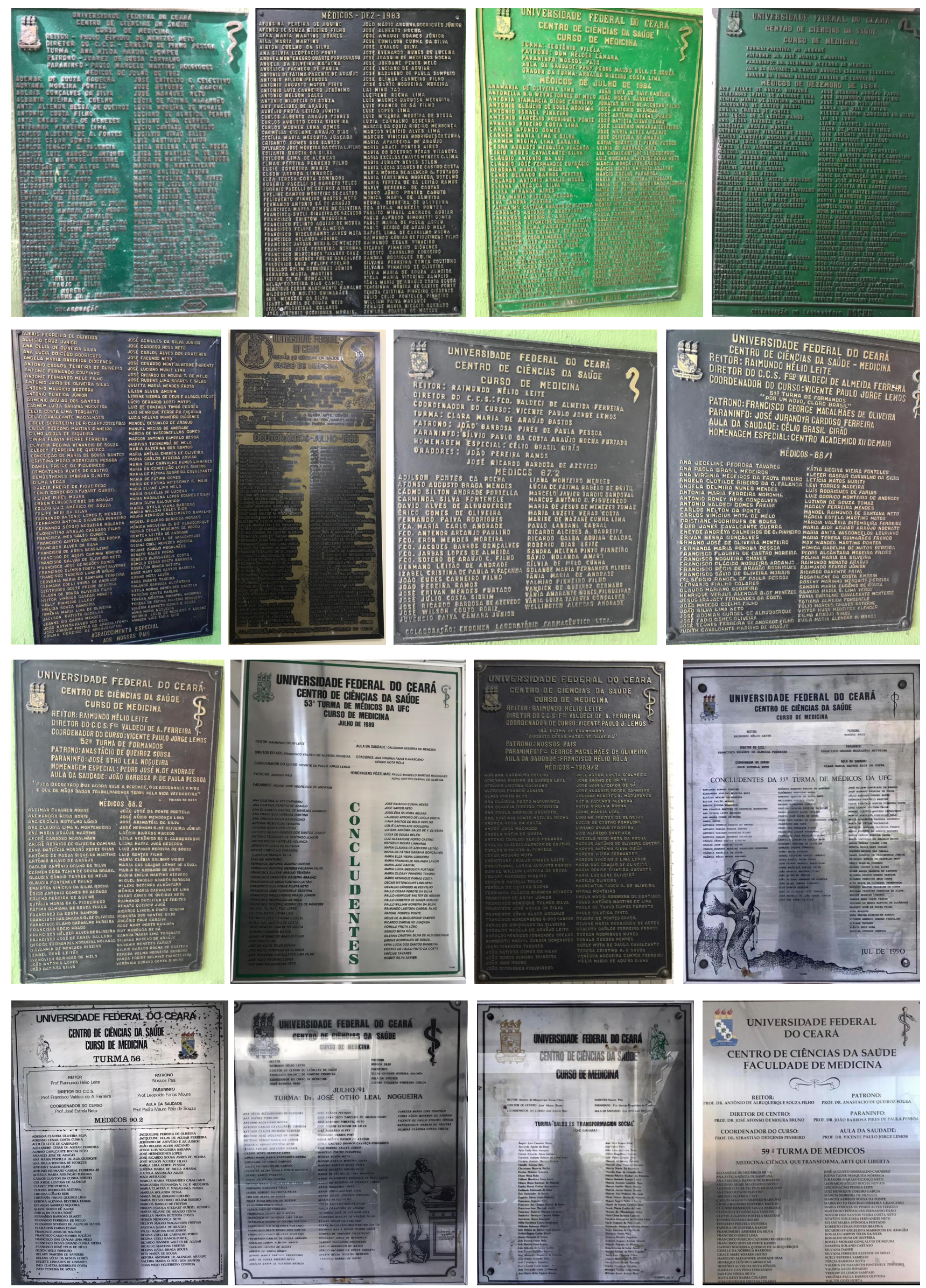
Figura 1. Placas de formatura da FM - UFC.
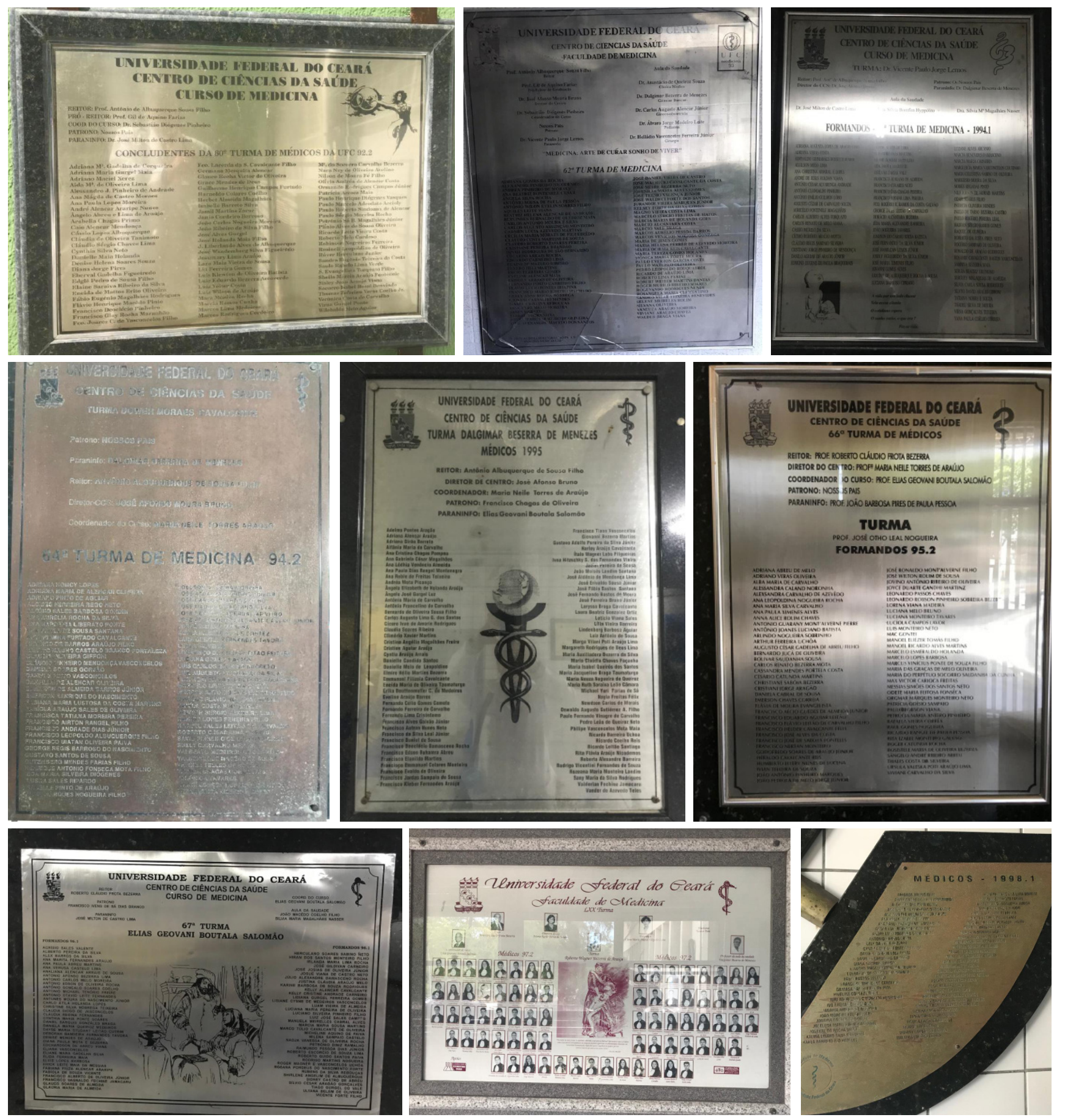

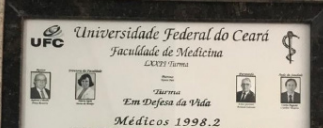

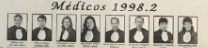

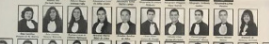

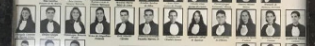

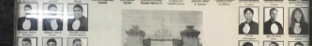
व.8 साता 802 6.8 $288=828$ $6.32=28$

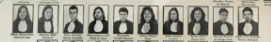

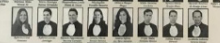
$8880=$

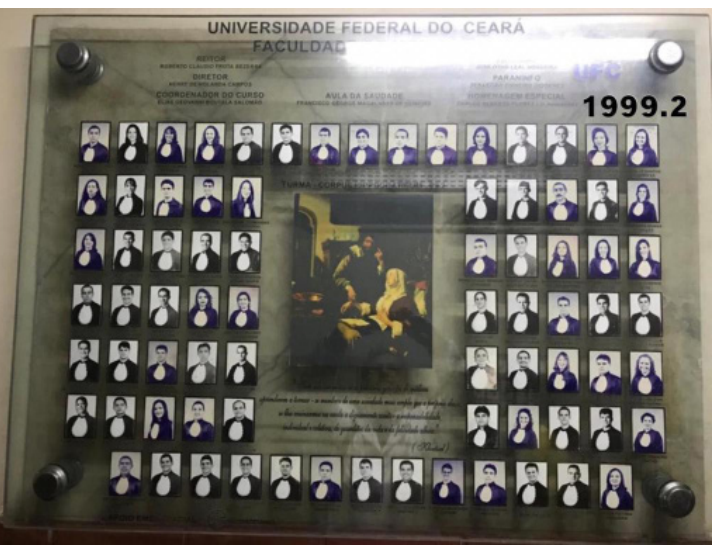


Figura 1. Placas de formatura da FM - UFC.
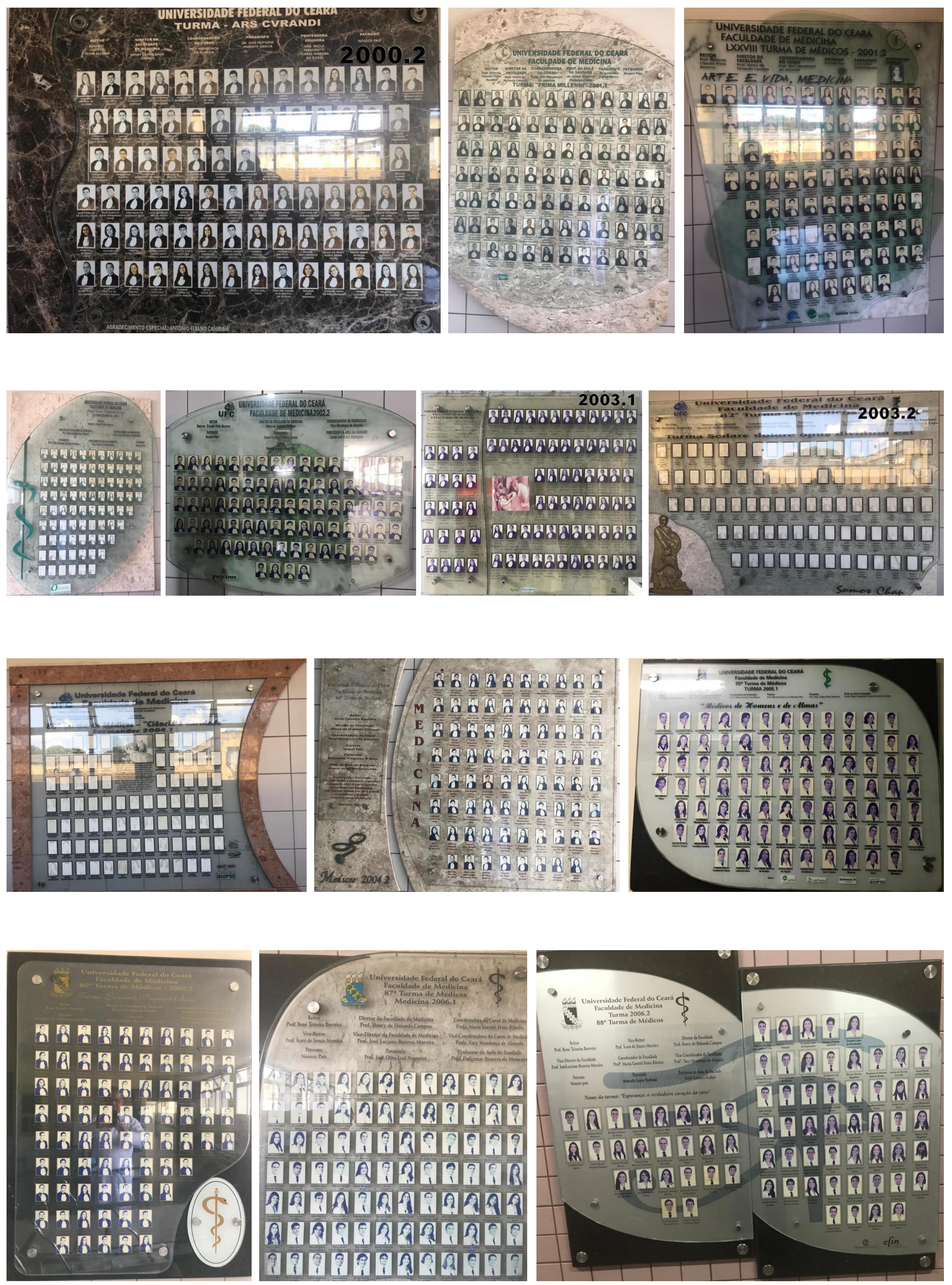
Figura 1. Placas de formatura da FM - UFC.
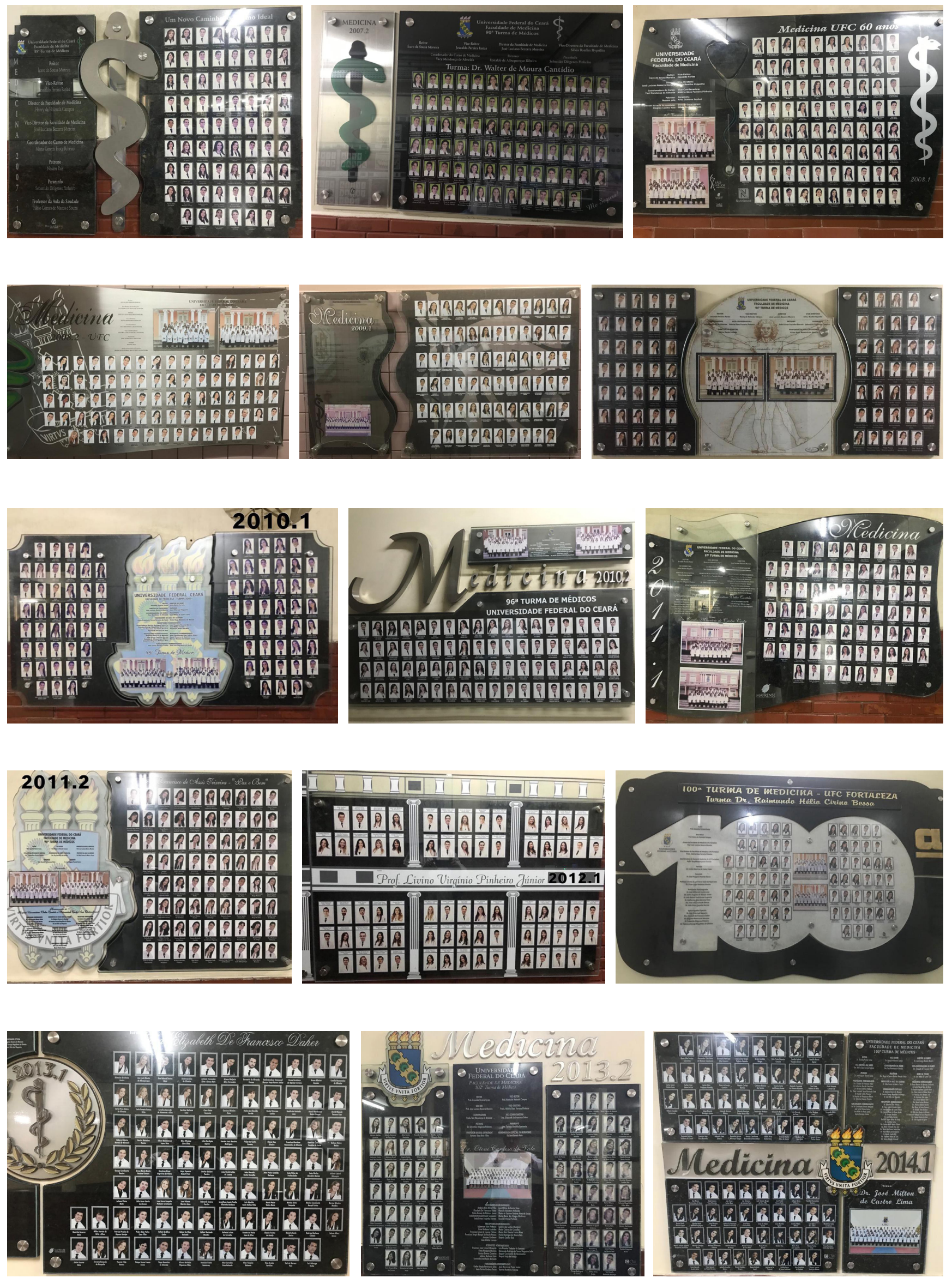
Figura 1. Placas de formatura da FM - UFC.
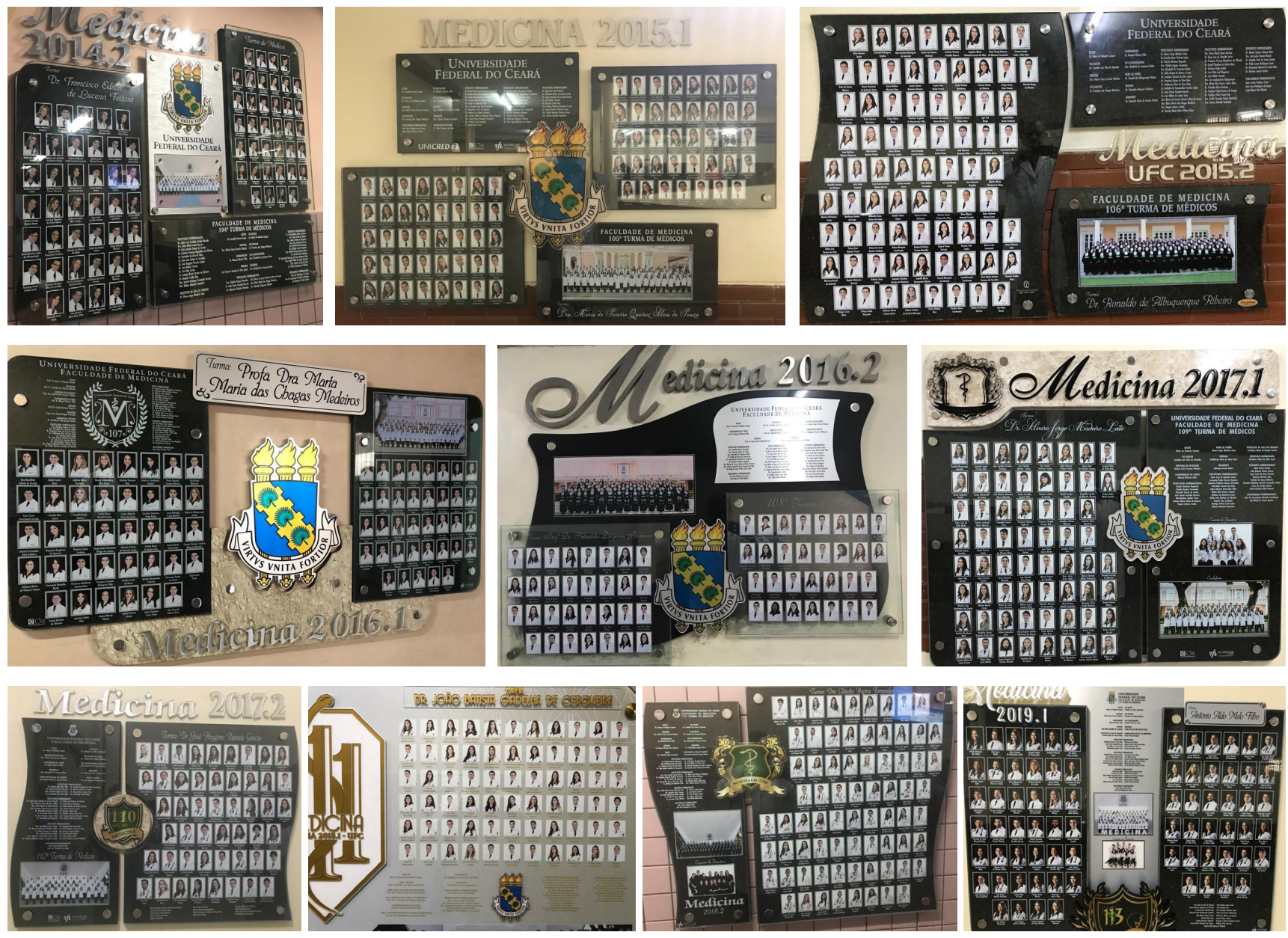

\section{REFERÊNCIAS}

1 Martins JM. Faculdade de Medicina da UFC: professores e médicos graduados. Fortaleza: Edições UFC; [date unknown]. volume 1 a 4.

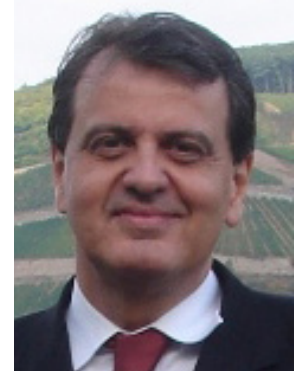

Dr. Ricardo Pereira Silva

Departamento de Medicina Clínica

Universidade Federal do Ceará

Como citar:

Silva RP. História das placas de formatura da Faculdade de Medicina da Universidade Federal do Ceará. Rev Med UFC. 2020 jan-mar;60(1):6-14. 